\title{
The Globalization of Respiratory CARE
}

Over the last few years, Respiratory CARe has been fortunate to receive an increasing number of manuscripts from authors outside the United States. There is no one reason for this change, but I thought it appropriate to reflect on a few factors that contribute to the ever-growing international appreciation, respect, and support of our science journal, Respiratory CARE.

It is no secret that there is worldwide recognition of the cost of healthcare. Many countries, including the United States, are struggling to balance the need for healthcare resources with their goal to maintain the world economy on a stable yet prosperous footing. Pulmonary diseases are now recognized as a major contributor to healthcare cost. Thus, more attention is focused on earlier detection of respiratory diseases and a thoughtful, efficient, and effective service delivery model to address the needs of these patients.

Over the years, the Journal has been on the cutting edge in publishing a wide range of approaches that leverage the virtual explosion of healthcare technology, while encouraging the use of evidence-based guidelines, protocols, and clinicians who have the appropriate knowledge and skills to apply what has been proven as clinically effective and cost-effective. The result is to optimize clinical outcomes, but also, especially in recent years, to assure that devices, treatment modalities, medications, and healthcare providers are utilized in a cost-effective fashion.

See the Original Study on Page 370

Respiratory CARE is not the exclusive turf of physicians, nurses, physiotherapists, or respiratory therapists. Respiratory care can be and is provided by all of these clinicians. As we know very well, one size never fits all. Our Journal exists to inform all who provide respiratory care services throughout the world. As illustrated by $\mathrm{Li}$ et $\mathrm{al}^{1}$ in this issue, the best approach for each country and

The author has disclosed no conflicts of interest.

Correspondence: Sam P Giordano MBA RRT FAARC, Executive Director, American Association for Respiratory Care, 9425 MacArthur Boulevard, Suite 100, Irving TX 75063-4706. E-mail: giordano@aarc.org.

DOI: $10.4187 /$ respcare. 01758 each healthcare system is to develop its own pathway to optimize respiratory care throughout the spectrum of care settings, acuities, and resources.

As we move forward into this century, we will continue to see more research effort devoted to the care of patients with respiratory disorders, regardless of whether they are chronic or acute, in the United States or elsewhere in the world. The burden of respiratory diseases is increasing, and our best hope to change this upward curve is to enable even more information sharing on best practices. The days of fragmented respiratory care must be put behind us, and to do that dedicated healthcare professionals must step up and choose to become the best, not only in rendering the wide range of clinical inventions, but also in promoting appropriate treatment that becomes a double-edged sword. But this particular double-edged sword, while it will cut both ways, will be different than the others. It will cut at respiratory disease and improve the respiratory quality of life for our patients worldwide. It will also cut at unnecessary care, unnecessary costs, and optimize the precious resources we have available for our patients. The healthcare professionals swinging this new sword may be called different names in different countries. But they must all master the medical evidence and practice guidelines in such a way as their utilization is universal and their benefit recognized and appreciated, not just by the medical communities, but also the world's policy makers.

The respiratory care provider community is a big tent and the only price of admission is comprehensive knowledge of the treatment of respiratory disorders. Please join the ranks of your colleagues around the world, regardless of discipline, to undertake critical thinking, use evidencebased practices, encourage and execute research, and, of course, think of our science journal, ResPiRatory CARE, when it comes time to publish your manuscript.

Sam P Giordano MBA RRT FAARC

Executive Director American Association for Respiratory Care Publisher, Respiratory CARE Irving, Texas

\section{REFERENCE}

1. Li J, Zhan QY, Liang ZA, Tu ML, Sun B, Yao XL, et al. Respiratory care practices and requirements for respiratory therapists in Beijing intensive care units. Respir Care 2012;57(3):370-376. 